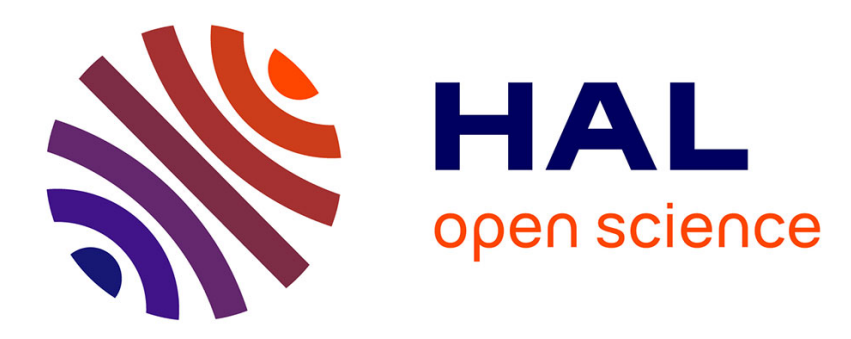

\title{
Indétermination et inefficacité des modèles de marchés de biens conditionnels à points de rationnement
}

\author{
Jean-Michel Courtault
}

\section{To cite this version:}

Jean-Michel Courtault. Indétermination et inefficacité des modèles de marchés de biens conditionnels à points de rationnement. Revue Economique, 1998, 49 (2), pp.367-392. halshs-00447608

\section{HAL Id: halshs-00447608 \\ https://shs.hal.science/halshs-00447608}

Submitted on 15 Jan 2010

HAL is a multi-disciplinary open access archive for the deposit and dissemination of scientific research documents, whether they are published or not. The documents may come from teaching and research institutions in France or abroad, or from public or private research centers.
L'archive ouverte pluridisciplinaire HAL, est destinée au dépôt et à la diffusion de documents scientifiques de niveau recherche, publiés ou non, émanant des établissements d'enseignement et de recherche français ou étrangers, des laboratoires publics ou privés. 


\title{
Persée
}

http://www.persee.fr

\section{Indétermination et inefficacité des modèles de marchés de biens conditionnels à points de rationnement}

\author{
Jean-Michel Courtault
}

Revue économique, Année 1998, Volume 49, Numéro 2

p. 367 - 392

Voir l'article en ligne

\section{Avertissement}

L'éditeur du site «PERSEE » - le Ministère de la jeunesse, de l'éducation nationale et de la recherche, Direction de l'enseignement supérieur, Sous-direction des bibliothèques et de la documentation - détient la propriété intellectuelle et les droits d'exploitation. A ce titre il est titulaire des droits d'auteur et du droit sui generis du producteur de bases de données sur ce site conformément à la loi n`98-536 du $1 \mathrm{er} \mathrm{juillet} 1998$ relative aux bases de données.

Les oeuvres reproduites sur le site «PERSEE » sont protégées par les dispositions générales du Code de la propriété intellectuelle.

Droits et devoirs des utilisateurs

Pour un usage strictement privé, la simple reproduction du contenu de ce site est libre.

Pour un usage scientifique ou pédagogique, à des fins de recherches, d'enseignement ou de communication excluant toute exploitation commerciale, la reproduction et la communication au public du contenu de ce site sont autorisées, sous réserve que celles-ci servent d'illustration, ne soient pas substantielles et ne soient pas expressément limitées (plans ou photographies). La mention Le Ministère de la jeunesse, de l'éducation nationale et de la recherche, Direction de l'enseignement supérieur, Sous-direction des bibliothèques et de la documentation sur chaque reproduction tirée du site est obligatoire ainsi que le nom de la revue et- lorsqu'ils sont indiqués - le nom de l'auteur et la référence du document reproduit.

Toute autre reproduction ou communication au public, intégrale ou substantielle du contenu de ce site, par quelque procédé que ce soit, de l'éditeur original de l'oeuvre, de l'auteur et de ses ayants droit.

La reproduction et l'exploitation des photographies et des plans, y compris à des fins commerciales, doivent être autorisés par l'éditeur du site, Le Ministère de la jeunesse, de l'éducation nationale et de la recherche, Direction de l'enseignement supérieur, Sous-direction des bibliothèques et de la documentation (voir http://www.sup.adc.education.fr/bib/ ). La source et les crédits devront toujours être mentionnés. 


\title{
Indétermination et inefficacité des modèles de marchés de biens conditionnels à points de rationnement
}

\author{
Jean-Michel Courtault*
}

\begin{abstract}
Nous montrons dans cet article, à la suite de Fischer [1972], qu'il est possible de formuler (dans le cas d'un bien physique unique) un problème économiquement équivalent au problème canonique du consommateur en situation d'incertitude et formellement isomorphe au problème du choix du consommateur en situation de rationnement. Ceci revient à généraliser au cas des marchés financiers incomplets le résultat d'Arrow [1964] sur l'équivalence entre le modèle des marchés de biens conditionnels complets et le modèle des marchés financiers complets et des marchés de biens au comptant. Nous étudions ensuite dans le cadre du modèle à points de rationnement le problème de lindétermination et de l'inefficacité de l'équilibre général.
\end{abstract}

\section{INDETERMINACY AND INEFFICIENCY IN A MODEL OF MARKETS WITH RATIONING}

Following Fischer [1972], we show in this paper (in the case of one physical good) the existence of a problem economically equivalent to the canonical consumer problem under uncertainty which is formally isomorphic to the problem of consumer choice under rationing. This amounts to generalise the result of Arrow [1964] on the equivalence of Arrow-Debreu model of complete contingent markets and the model of complete financial markets with spot markets. We then use the model of rationing to study the problem of indetermination and inefficiency of general equilibrium.

Classification JEL : D5, D8

\section{INTRODUCTION}

D'après I. Maes [1991], c'est à l'instigation de L. Robbins que J. Hicks [1935] a cherché à introduire la théorie des choix dans la théorie monétaire après l'avoir fait brillamment, conjointement avec R. G. D. Allen [1934], pour la théorie du consommateur. Cela contribuait à réaliser le programme de recherche défini par Robbins [1932] où il définissait la science économique comme « la science qui étudie le comportement humain en tant que relation entre des fins et des moyens rares à usages alternatifs ».

\footnotetext{
* CRESE - Université de Franche-Comté, Avenue de l'Observatoire, 25030 Besançon.

Je remercie les deux rapporteurs anonymes de la Revue économique ainsi que J.-M. Rousseau pour leurs commentaires.
} 
Les principaux éléments de la théorie du portefeuille moderne de J. Tobin [1958] et H. Markowitz [1959] se trouvent en substance dans l'article séminal de Hicks [1935] : un individu cherchant le portefeuille qui maximise une fonction d'utilité de la moyenne et de l'écart type du rendement du portefeuille sous une contrainte de richesse. Il devenait alors possible de faire appel, compte tenu de la ressemblance entre ce programme et celui du consommateur, aux concepts d'effets de substitution et d'effets richesse pour comprendre la détermination de l'allocation de la richesse initiale entre les différents actifs financiers et monétaires, les anticipations en matière de risque et de rendement jouant un rôle analogue aux prix dans la théorie du consommateur ${ }^{1}$.

Il y a eu un grand nombre de travaux qui ont cherché à exploiter l'analogie entre les théories de la demande de biens et d'actifs financiers. Tout d'abord, un certain nombre d'articles ont cherché à le faire dans le cadre du modèle Moyenne-Variance, e.g. S. Royama et K. Hamada [1967], G. Bierwag et M. Grove [1968], H. Levy [1973] et M. Morishima [1973]. Cependant, bien que l'analyse Moyenne-Variance puisse être considérée comme une approximation $v^{\text {valable }}{ }^{2}$, de telles analyses ont nécessairement une portée limitée. C'est pourquoi de nombreux auteurs ont utilisé les outils analytiques de la théorie de la demande dans des conditions plus générales que celles du modèle MoyenneVariance, comme par exemple S.-C. Kolm [1966], A. Sandmo [1969], et A. Dalal [1983].

Plus récemment, He et Pearson [1991] ont cherché à savoir sous quelles conditions un programme dynamique de choix de consommation et de portefeuille est équivalent à un programme statique de choix d'un plan de consommation conditionnel et quelle est alors la forme du problème statique. Ils montrent qu'il existe, dans un système incomplet de marchés financiers, une mesure martingale équivalente, ou un système de prix d'états implicites pour lequel un programme dynamique de choix de consommation et de portefeuille est équivalent à un programme statique de choix d'un plan de consommation conditionnel. Nous montrerons le lien qui existe entre l'approche développée par ces auteurs et celle de Fischer [1972] et en particulier comment celle-ci peut nous permettre de calculer le système de prix d'états implicites de He et Pearson.

L'objet du présent article est de chercher s'il existe, au-delà de la simple analogie, un isomorphisme entre les problèmes de choix auxquels sont confrontés un investisseur et un consommateur. En effet, il semble indispensable de montrer qu'il existe un tel isomorphisme entre ces théories sans quoi il serait impossible d'appliquer les concepts (notamment ceux d'effet de substitution et d'effet revenu) qui permettent de rendre compte de façon satisfaisante du comportement du consommateur au problème du choix de portefeuille sans dénaturer de manière injustifiée la réalité des comportements financiers.

D'une manière générale, les microéconomistes ont souvent tendance à exprimer les phénomènes qu'ils cherchent à expliquer dans le cadre familier de la théorie du consommateur. L'intérêt de formaliser un problème économique de telle sorte que sa structure soit isomorphe à celle de la théorie du consommateur vient de ce que les résultats qui ont été établis par cette dernière peuvent être

1. Voir I. Maes [1986], p. 408-410.

2. Voir P. Samuelson [1970] et S. Tsiang [1972]. 
transposés pour rendre compte d'autres comportements ${ }^{1}$. On estime, en général, que cette identité dans la structure formelle des modèles ne doit pas être obtenue au prix d'une distorsion de la réalité du phénomène à étudier, la modélisation d'un phénomène étant censé être une représentation intellectuelle de ce phénomène ; elle doit être autant que faire se peut aussi réaliste que possible. Nous verrons cependant, en conclusion, comment l'existence d'un isomorphisme peut justifier dans certains cas l'utilisation de modèles irréalistes.

Nous montrerons, en nous appuyant sur les travaux de S. Fischer [1972] et de J. Ohlson [1987], qu'il est possible de déterminer un programme équivalent à celui de la sélection de portefeuille sous sa forme canonique parfaitement isomorphe à celui du choix du consommateur en situation de rationnement : nous aurons ainsi montré qu'il est possible de bénéficier de la simplicité formelle de la théorie du consommateur sans toutefois renoncer au réalisme de la théorie traditionnelle du portefeuille. S. Fischer utilisait cet isomorphisme pour étudier les propriétés des demandes d'actifs financiers (notamment les relations de Slutsky), nous l'utilisons pour étudier les problèmes d'indétermination et d'inefficacité des marchés financiers incomplets.

\section{LA VERSION CANONIQUE DU PROBLÈME DE LA SÉLECTION DE PORTEFEUILLE ${ }^{2}$}

Dans le modèle standard du portefeuille, l'activité économique n'a lieu qu'à deux dates seulement : à l'instant zéro («aujourd'hui ») et à l'instant un ( « demain »). L'agent économique prend des décisions aujourd'hui relativement à sa consommation courante et à ses investissements qui se traduisent par un niveau incertain de la richesse pour le futur. Les décisions de l'agent ne concernent que la quantité de bien $\mathrm{C}_{0}$ qu'il consommera au cours de la première période (de l'instant 0 à l'instant 1) ainsi que l'importance et la composition du portefeuille qu'il détiendra jusqu'à la date 1 , date à laquelle son portefeuille sera liquidé pour faire face à ses besoins de consommation de la seconde période (de la date 1 jusqu'à sa mort). Par souci de simplification, l'analyse fait abstraction des opportunités d'investissement à la date 1 , ainsi que des possibilités de diversification de la consommation aux deux périodes. Le prix du bien de consommation de la première et de la seconde période peut être fixé égal à un puisque, d'une part, la richesse initiale de l'agent peut être exprimée en unités de bien de consommation en divisant la richesse nominale par le prix du bien en première période et puisque, d'autre part, les rendements des actifs peuvent toujours être exprimés relativement au prix du bien en seconde période.

La valeur liquidative du portefeuille étant incertaine à l'instant 1, la consommation future $\mathrm{C}_{s}$ l'est également. L'incertitude dans l'économie est caractérisée

1. La valeur heuristique d'un tel postulat nous semble bien établie du moins si l'on en juge d'après la valeur scientifique du corps de connaissances qu'il a permis de constituer. Voir P.-A. Chiappori [1990] et A. Deaton et J. Muellbauer [1980].

2. Pour cette section, nous avons utilisé les travaux de J. Ingersoll ([1987], chap. 2 et 3), R. Jarrow ([1988], chap. 8) et J. Ohlson ([1987], chap. 2). 
par un ensemble d'états de la nature possibles, que nous supposerons être en nombre fini $\mathrm{S}$, n'importe lequel de ces états pouvant survenir. Un état de la nature $s(s=1, \ldots, S)$ est une description complète des conditions prévalant dans l'économie à la date 1 .

À la date 0 , le consommateur dispose d'une certaine richesse initiale qui doit être affectée à la consommation initiale $\mathrm{C}_{0}$ et à l'achat d'un portefeuille d'actifs $\boldsymbol{x}^{\prime}$ $=\left(x_{1}, \ldots, x_{N}\right)$ aux prix $p_{0}$ égal à 1 , et $\boldsymbol{q}^{\prime}=\left(q_{1}, \ldots, q_{\mathrm{N}}\right)$ strictement positifs, respectivement. Les composantes du portefeuille d'actifs $\boldsymbol{x}$ peuvent être positives ou négatives : une valeur positive de $x_{\mathrm{i}}$ s'interprète comme une position longue sur l'actif $i$, si bien que l'on reçoit le paiement correspondant si l'état $s$ se réalise ; tandis qu'une valeur négative de $x_{\mathfrak{i}}$ s'interprète comme une vente à découvert de l'actif $i$, si bien que l'on doit donner le paiement correspondant si l'état $s$ se réalise.

Par conséquent, un actif peut être interprété comme une fonction de l'espace des états de la nature $\Omega$ dans $\mathbb{R}$ :

$$
y_{i}: \Omega \rightarrow \mathbb{R} \quad i=1, \ldots, \mathrm{N}
$$

La matrice des paiements de l'ensemble des actifs s'écrit alors :

$$
\mathrm{Y}=\left[\begin{array}{ccc}
y_{\lambda 1} & \ldots & y_{1 N} \\
\vdots & & \vdots \\
y_{S 1} & \ldots & y_{S N}
\end{array}\right]
$$

Cette matrice $(S \times N)$ résume les caractéristiques des paiements offerts par un ensemble donné d'actifs. Elle donne les paiements de chacun des $\mathrm{N}$ actifs dans chacun des $\mathrm{S}$ états de la nature : chacune des colonnes de cette matrice de paiements représente un actif différent et chaque ligne donne les paiements pour un état donné de la nature de chacun des actifs.

On dit d'un portefeuille $\boldsymbol{x}^{1}$ qu'il est duplicable s'il existe un portefeuille $\boldsymbol{x}^{2}$ différent du premier mais dont le coût est identique tel que l'on ait :

$$
\mathbf{Y} \boldsymbol{x}^{2}=\mathbf{Y} \boldsymbol{x}^{1}
$$

C'est-à-dire qu'il existe un portefeuille différent dont le rendement est identique dans tous les états de la nature. S'il existe une paire de portefeuilles duplicables $\boldsymbol{x}^{1}$ et $\boldsymbol{x}^{2}$, alors tout portefeuille $\boldsymbol{x}$ est duplicable puisque $\boldsymbol{x}+\left(\boldsymbol{x}^{1}-\boldsymbol{x}^{2}\right)$ le duplique. Il est clair que de tels portefeuilles ne servent aucune fonction économique et sont par conséquent inutiles. Ceci n'est vrai toutefois que dans le cadre du modèle envisagé où il n'existe ni coût de transaction, ni indice de liquidité. En effet, l'existence des marchés dérivés attestent, au moins en partie, de l'intérêt économique des actifs redondants.

La duplicabilité des portefeuilles peut être interprétée comme la conséquence de la redondance de certains actifs. D'après la définition de la duplicabilité, il s'ensuit qu'au moins un des actifs est redondant s'il existe un portefeuille dont le coût est nul ainsi que le paiement

$$
\boldsymbol{x} / \mathbf{Y} \boldsymbol{x}=0 \quad q^{\prime} \boldsymbol{x}=0 \quad \boldsymbol{x} \neq 0
$$

On sait qu'il existe une solution non nulle à ce système homogène de $\mathrm{S}+\mathrm{l}$ équations linéaires à $\mathrm{N}$ inconnues si et seulement si les vecteurs lignes de la 
matrice $\hat{\mathbf{Y}}=\left[\mathbf{Y}^{\prime}, \boldsymbol{q}\right]$ sont linéairement dépendants ${ }^{1}$. En d'autres termes, il n'existe pas d'actif redondant si le rang de la matrice $\hat{\mathbf{Y}}$ est $\mathbf{N}$.

Nous supposerons par la suite qu'il n'existe pas d'actif redondant. Si cette rationalisation n'était pas effectuée, il deviendrait inutile de se poser la question de l'unicité du portefeuille optimal.

Une opportunité d'arbitrage signifie qu'un individu peut obtenir quelque chose à partir de rien. Dans cette situation, il est intuitif qu'il n'existe pas de solution optimale au problème de choix de portefeuille puisqu'un agent peut toujours accroître le niveau de son utilité. En effet, supposons l'existence d'un portefeuille $\boldsymbol{x}^{1}$ dominant un portefeuille $\boldsymbol{x}^{2}$, c'est-à-dire un portefeuille remplissant les conditions suivantes

$$
\begin{aligned}
& q^{\prime} x^{1}=q^{\prime} x^{2} \\
& Y x^{1} \geqslant Y x^{2}
\end{aligned}
$$

Le portefeuille $\boldsymbol{x}^{1}$ étant bien supérieur à $x^{2}$ puisqu'il ne coûte pas plus cher à acquérir et qu'il rapporte plus quelles que soient les circontances ${ }^{2}$. Il est clair que tout individu possédant des préférences monotones croissantes préfère $x^{1}$ à $\boldsymbol{x}^{2}$. L'existence d'un tel portefeuille implique qu'il n'existe pas de portefeuille optimal. En effet, tout portefeuille $x$ est dominé par le portefeuille $x+\gamma\left(x^{1}-x^{2}\right)$ pour tout $\gamma>0$.

La définition suivante de l'absence d'opportunités d'arbitrage découle de la définition que nous avons donnée de la dominance d'un portefeuille. On dit qu'il n'y a pas d'opportunités d'arbitrage s'il n'existe pas de portefeuille $x$ tel que

$$
\boldsymbol{q}^{\prime} \boldsymbol{x} \leqslant 0, \quad \mathbf{Y} \boldsymbol{x} \geqq 0
$$

avec au moins une inégalité stricte parmi les $S+1$ équations.

Il est important de noter que la définition ne fait pas intervenir les probabilités des états de la nature mais seulement les possibilités. Par conséquent, la présence ou l'absence d'opportunités d'arbitrage n'est pas un phénomène subjectif puisque l'ensemble des états possibles de la nature est une donnée du problème et seront considérées comme telles par tous les participants. La seule restriction quant aux croyances est que chaque agent doit considérer que chaque état de la nature a une probabilité non nulle de survenir. Si un investisseur quelconque pensait qu'un certain état de la nature soit impossible, il serait prêt à vendre à découvert pour rien un portefeuille qui ne rapporterait rien dans tous les autres états de la nature. Tout autre investisseur qui penserait que cet état est possible considérerait le don de cet investisseur comme une opportunité d'arbitrage.

On dit que les marchés sont complets si l'on peut obtenir une configuration quelconque de richesse à partir des actifs disponibles, i.e.

$$
\forall \bar{w} \in \mathbb{R}^{S}, \exists \boldsymbol{x} \in \mathbb{R}^{\mathrm{N}} / \mathbf{Y} \boldsymbol{x}=\overline{\boldsymbol{w}}
$$

Il existe une solution à ce système d'équations si, et seulement si, la matrice des paiements $\mathbf{Y}$ est de plein rang, c'est-à-dire si $\operatorname{dim}\left\{\boldsymbol{y}_{\boldsymbol{i}}\right\}_{i}=\mathrm{S}$. En effet, si la matrice fondamentale $\mathbf{Y}$ est de plein rang la matrice complète $(\mathbf{Y}, \bar{w})$ l'est également et,

1. Voir L. Koulikov [1979], proposition 3.7, p. 187.

2. Si $\boldsymbol{x}^{1}$ coûtait moins cher que $\boldsymbol{x}^{2}$, il suffirait d'accroître son échelle pour restaurer l'égalité. 
par la règle des rangs ${ }^{1}$, il existe toujours une solution au système d'équations précédent. Les marchés sont incomplets si la matrice des paiements n'est pas de plein rang. On aura donc des marchés incomplets lorsque le nombre d'actifs est inférieur au nombre d'états de la nature et des marchés complets lorsqu'il y a autant d'actifs indépendants que d'états de la nature. Dans le cas où il y a davantage d'actifs que d'états, il y a soit des opportunités d'arbitrage, soit des actifs qui sont redondants, ce qui a été éliminé par hypothèse.

La consommation de l'individu en seconde période résulte à la fois du rendement de son portefeuille $\boldsymbol{x}$ dans l'état $s$ et de ses dotations $e_{s}$. La consommation future est donc donnée par la formule :

$$
\mathrm{C}_{s} \leqslant \sum_{j=1}^{\mathrm{N}} x_{j} y_{j s}+e_{s} \quad s=1, \ldots, \mathrm{S}
$$

En combinant les préférences d'un individu avec ses ressources et les possibilités offertes par le marché, on aboutit à la définition suivante du problème du portefeuille sous sa forme canonique. Dans le problème standard, l'agent cherche à résoudre :

PROGRAMME I

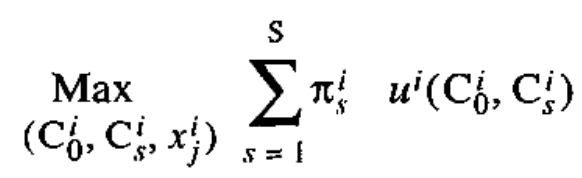

sous les contraintes :

$$
\begin{gathered}
\mathrm{C}_{0}^{i}+\sum_{j=1}^{\mathrm{N}} q_{j} x_{j}^{i} \leqslant e_{0}^{i} \\
\mathrm{C}_{s}^{i} \leqslant \sum_{j=1}^{\mathrm{N}} x_{j}^{i} y_{j s}+e_{s}^{i} \quad s=1, \ldots, \mathrm{S}
\end{gathered}
$$

Le problème étant posé, il est naturel de chercher les conditions qui assurent l'existence et l'unicité d'une solution optimale. Outre les hypothèses de monotonicité et de stricte concavité de la fonction d'utilité, l'existence d'un optimum suppose également l'absence d'opportunités d'arbitrage. En utilisant le lemme de Farkas, on peut montrer que cette condition entraîne l'existence de prix d'états strictement positifs.

La question alors se pose de savoir s'il existe un vecteur $\boldsymbol{q}$ tel que les caractéristiques du marché $(\mathbf{Y}, \boldsymbol{q})$ excluent les possibilités d'arbitrage ? Le théorème suivant permet de répondre affirmativement à cette question.

THÉORÈME 1 (S. Ross [1978]). Il existe un vecteur de prix d'états positifs $p$ tel que $\boldsymbol{q}^{\prime}=\boldsymbol{p}^{\prime} \mathbf{Y}$ si, et seulement si, les caractéristiques du marché $(\mathbf{Y}, \boldsymbol{q})$ excluent toute possibilité d'arbitrage ${ }^{2}$.

1. Voir L. Koulikov [1979], théorèmes $2.12,2.13$ et 2.14, p. 177-178.

2. On peut trouver la démonstration dans J. Ingersoll [1987], p. 54-58 ou dans l'ouvrage de E. Malinvaud [1993], p. 199-201. 
La condition d'absence d'opportunités d'arbitrage implique donc qu'il existe un vecteur de prix d'états positifs $\boldsymbol{p}^{\prime}=\left(p_{1}, \ldots, p_{s}\right)$ tel que le prix de tout actif est donné par:

$$
q_{i}=\sum_{s=1}^{\mathrm{s}} p_{s} y_{i s}
$$

Les deux caractéristiques importantes de ce théorème sont la positivité des prix d'états et la linéarité de la formule donnant le prix des actifs. C'est la propriété de positivité qui assure l'absence d'opportunités d'arbitrage : si le prix d'une unité de consommation dans l'état $s p_{s}$ était négatif ou nul, il serait possible de consommer sans avoir à payer et l'on ferait même un profit. La propriété de linéarité implique que deux unités de consommation dans l'état $s$ valent deux fois plus cher qu'une seule (il n'y a pas d'effets d'échelle); de plus, la valeur d'une unité de consommation dans l'état $s$ ne dépend pas des paiements dans les autres états de la nature. Enfin, si la relation n'était pas linéaire, il serait alors profitable de constituer des portefeuilles qui auraient une valeur plus ou moins grande que les actifs qui les constituent.

Le vecteur des prix d'états n'est pas nécessairement unique; si le rang de $\mathbf{Y}$ est inférieur à $S$, alors il existe au moins deux vecteurs $\boldsymbol{p}$ et $\boldsymbol{p}^{*}$ tels que $\boldsymbol{q}^{\prime}=\boldsymbol{p}^{\prime}$ $\mathbf{Y}=\boldsymbol{p}^{* \prime} \mathbf{Y}$ bien que $\boldsymbol{p} \neq \boldsymbol{p}^{*}$ et $\boldsymbol{p}, \boldsymbol{p}^{*}>0$. Si en effet le nombre des actifs non redondants $\mathrm{N}$ est inférieur au nombre d'états de la nature $\mathrm{S}$, alors l'absence d'opportunités d'arbitrage n'implique que $\mathrm{N}$ restrictions linéaires sur le vecteur des prix d'états et laisse $\mathrm{S}-\mathrm{N}$ degrés de liberté. De plus, l'existence d'un vecteur $\boldsymbol{p}$ dont certaines composantes sont strictement négatives tel que $\boldsymbol{q}^{\prime}=\boldsymbol{p}^{\prime} \mathbf{Y}$ n'implique pas l'existence d'opportunités d'arbitrage sauf si bien sûr $\boldsymbol{p}$ est unique. Le théorème 1 nous montre simplement que l'absence d'opportunités d'arbitrage est impliquée par l'existence d'au moins un vecteur positif $\boldsymbol{p}$ et que c'est aussi une condition nécessaire. C'est seulement dans le cas où il existe autant d'actifs indépendants que d'états de la nature que l'on est sûr que le vecteur des prix d'états est défini de manière unique.

Il est maintenant possible de déterminer les conditions nécessaires et suffisantes qui garantissent l'existence et l'unicité du portefeuille optimal.

THÉORÈME 2. Soit $\mathrm{U}\left(\mathrm{C}_{0}, \mathbf{C}\right)$ une fonction d'utilité croissante et strictement concave ; le problème canonique admet une solution unique $\left(\mathrm{C}_{0}, \boldsymbol{x}\right)$ si et seulement si il existe ni opportunités d'arbitrage ni portefeuille redondant ${ }^{1}$.

On peut donner l'intuition de ce résultat en utilisant un théorème de Luenberger d'après lequel tout programme d'optimisation à contraintes multiples peut se ramener à un programme d'optimisation à contrainte unique ${ }^{2}$. Le programme du consommateur à contraintes budgétaires multiples (Programme I) peut se ramener à un programme à contrainte budgétaire unique (Programme I') :

1. Voir la démonstration dans J. E. Ingersoll [1987], p. 67-68 ou dans J. A. Ohlson [1987], p. 17-18.

2. Voir Luenberger [1995], p. 156-160. 
PROGRAMME I' :

$$
\operatorname{Max}_{\left(\mathrm{C}_{0}^{i}, \mathrm{C}_{s}^{i}\right)} \sum_{s=1}^{\mathrm{S}} \pi_{s}^{i} \quad u^{i}\left(\mathrm{C}_{0}^{i}, \mathrm{C}_{s}^{i}\right)
$$

sous la contrainte :

$$
\lambda_{0} \mathrm{C}_{0}^{i}+\sum_{s=1}^{\mathrm{S}} \lambda_{s} \mathrm{C}_{s}^{i} \leqslant \lambda_{0} e_{0}^{i}-\sum_{j=1}^{\mathrm{N}} x_{j}^{i}\left(\lambda_{0} q_{j}-\sum_{s=1}^{\mathrm{S}} \lambda_{s} y_{j s}\right)+\sum_{s=1}^{\mathrm{S}} \lambda_{s} e_{s}^{i}
$$

où les $\lambda_{s}(s=0, \ldots, S)$ peuvent être interprétés comme des prix relatifs et en tant que tels sont définis à un coefficient multiplicatif (strictement positif) près. $\lambda_{0}$ peut donc être interprété comme le prix du bien en première période (que l'on prend comme numéraire en fixant $\lambda_{0}=1$ ) et $\lambda_{\mathrm{s}}$ peut s'interpréter comme le prix conditionnel du bien dans l'état $s$.

On peut donner l'intuition de cette équivalence en montrant que le lagrangien associé au programme I peut être écrit de manière identique au lagrangien associé au programme I'. En effet, le lagrangien du programme I peut s'écrire :

$$
\begin{aligned}
\mathrm{L}_{\mathbf{I}}=\sum_{s=1}^{\mathrm{S}} \pi_{s}^{i} u^{i}\left(\mathrm{C}_{0}^{i}, \mathrm{C}_{s}^{i}\right)+\mu_{0}\left(e_{0}^{i}-\mathrm{C}_{0}^{i}-\sum_{j=1}^{\mathrm{N}} q_{j} x_{j}^{i}\right)+ & \\
& \sum_{s=1}^{\mathrm{S}} \mu_{s}\left(e_{s}^{i}-\mathrm{C}_{s}^{i}+\sum_{j=1}^{\mathrm{N}} y_{j s} x_{j}^{i}\right)
\end{aligned}
$$

En supposant que $\mu_{0}>0$, on peut réécrire $\mathrm{L}_{\mathrm{I}}$ de la manière suivante :

$$
\begin{aligned}
\mathrm{L}_{\mathrm{I}}=\sum_{s=1}^{\mathrm{S}} \pi_{s}^{i} u^{i}\left(\mathrm{C}_{0}^{i}, \mathrm{C}_{s}^{i}\right)+\mu_{0}\left(e_{0}^{i}-\mathrm{C}_{0}^{i}-\sum_{j=1}^{\mathrm{N}} q_{j} x_{j}^{i}+\right. & \\
& \left.\sum_{s=1}^{\mathrm{S}} \frac{\mu_{s}}{\mu_{0}}\left(e_{s}^{i}-\mathrm{C}_{s}^{i}+\sum_{j=1}^{\mathrm{N}} y_{j s} x_{j}^{i}\right)\right)
\end{aligned}
$$

ce qui est identique au lagrangien associé au programme I' une fois que l'on a posé $\lambda_{0}=1$. Les lagrangiens des deux programmes étant identiques, il s'ensuit que les solutions des deux programmes sont également identiques et que les programmes (I) et (I') sont équivalents.

La contrainte budgétaire unique du programme I' indique que s'il n'est pas possible de vendre à découvert l'actif $j\left(x_{j}^{i} \geqslant 0\right)$ il est alors nécessaire que :

$$
\lambda_{0} q_{j}-\sum_{s=1}^{s} \lambda_{s} y_{j s} \geqslant 0
$$

En effet, si cette inégalité n'était pas respectée, il serait possible d'accroître indéfiniment le membre de droite de la contrainte inégalité (représentant la valeur actualisée des ressources de l'agent) en achetant une quantité infinie d'actif $j$ (ce qui suppose que l'on peut vendre à découvert en quantité illimitée au moins un autre actif). Il serait alors possible d'acheter une quantité illimitée de biens de consommation et le programme du consommateur n'aurait pas de solution. Par 
ailleurs, si l'inégalité est stricte, le consommateur n'achètera pas d'actif $j$. En effet, lorsque l'inégalité est stricte, le consommateur a toujours la possibilité d'augmenter sa richesse actualisée en diminuant la quantité d'actif $j$. Il s'ensuit également que si le consommateur a une quantité non nulle d'actif $j$, l'inégalité est en fait une égalité.

Dans le cas que nous avons envisagé, il est possible d'acheter ou de vendre à découvert n'importe quel actif en quantité illimitée. Le programme du consommateur n'a alors de solution que si la relation suivante est respectée :

$$
\lambda_{0} q_{j}-\sum_{s=1}^{\mathrm{s}} \lambda_{s} y_{j s}=0 \quad j=1, \ldots, \mathrm{N}
$$

Si la relation d'arbitrage (10) est respectée, il s'ensuit qu'il existe une solution au programme I' (ainsi qu'au programme I).

Les conditions d'optimalité nous assure que la relation $\left(10^{\prime}\right)$ sera respectée. Par conséquent à l'optimum, la contrainte budgétaire du programme (I') se simplifie de la façon suivante :

$$
\lambda_{0} \mathrm{C}_{0}^{i}+\sum_{s=1}^{\mathrm{s}} \lambda_{s} \mathrm{C}_{s}^{i} \leqslant \lambda_{0} e_{0}^{i}+\sum_{s=1}^{\mathrm{s}} \lambda_{s} e_{s}^{i}
$$

On retrouve ainsi le résultat de He et Pearson [1991] suivant lesquels il existe un système de prix implicites tel que le programme dynamique à contraintes multiples d'un consommateur en situation d'incertitude peut se ramener à un programme statique à contrainte unique. Les prix d'états implicites étant égaux à la valeur des multiplicateurs de Lagrange associés aux contraintes du programme I.

\section{LA FORMULATION DE S. FISCHER [1972]}

Il est possible de développer un problème équivalent au problème standard du portefeuille, le problème du choix des biens contingents sous contrainte de rationnement qui est basé sur l'idée que les actifs financiers ne sont que les moyens qui permettent aux agents d'acquérir une certaine configuration de consommation future. Dans cette optique, $\left(\mathrm{C}_{0}, \mathrm{C}\right)$ devrait être considéré comme le vecteur de décisions sans qu'il soit besoin de se référer explicitement aux actifs qui permettent d'atteindre cette configuration particulière comme dans le modèle standard où le vecteur de décision est $\left(\mathrm{C}_{0} \boldsymbol{x}\right)$. Nous développerons cette équivalence dans le cas d'un bien de consommation unique puis nous donnerons un exemple numérique.

\section{Analyse générale}

Le théorème 1 montre qu'il est très facile de construire un vecteur $q$ qui exclut toute opportunité d'arbitrage en prenant pour vecteur de prix des actifs $\boldsymbol{p}^{\prime} \mathbf{Y}$ avec $\boldsymbol{p}$ vecteur positif quelconque. En revanche, étant donné les caractérisques du marché financier $(\mathbf{Y}, \boldsymbol{q})$ il est beaucoup plus difficile de déterminer un 
vecteur positif $\boldsymbol{p}$ ou de démontrer qu'il n'en existe pas lorsque $\mathbf{Y}$ est une matrice avec un grand nombre de lignes et de colonnes. Cependant, la non-unicité de $p$ n'empêche pas cette représentation d'être utile et théoriquement significative. En effet, les actifs sont des moyens permettant d'atteindre une fin : la consommation future. L'analyse peut donc être tout aussi bien menée en termes de $\mathbf{C}$ qu'en termes de $\boldsymbol{x}$. Toutefois, en l'absence d'un système complet de marchés financiers, il n'est pas possible à un individu d'atteindre toutes les configurations de consommation imaginables. On doit donc ajouter à la contrainte budgétaire traditionnelle (par rapport au modèle Arrow-Debreu) un certain nombre de contraintes supplémentaires qui correspondent à l'incomplétude des marchés dans le problème canonique. Le programme que le consommateur doit résoudre s'écrit alors :

PROGRAMME (II)

$$
\operatorname{Max}_{\left(\mathrm{C}_{0}^{i}, \mathbf{C}^{i}\right)} \sum_{s=1}^{\mathrm{s}} \pi_{s}^{i} \quad u^{i}\left(\mathrm{C}_{0}^{i}, \mathrm{C}_{s}^{i}\right)
$$

s.c.

$$
\begin{gathered}
\mathrm{C}_{0}^{i}+\sum_{s=1}^{\mathrm{S}} p_{s} \mathrm{C}_{s}^{i} \leqslant e_{0}^{i}+\sum_{s=1}^{\mathrm{S}} p_{s}\left(e_{s}^{i}+\sum_{j=1}^{\mathrm{N}} y_{j s} \bar{x}_{j}^{i}\right) \\
\sum_{s=1}^{\mathrm{S}} p_{k s} \mathrm{C}_{s}^{i}=\varepsilon_{k}^{i} \quad k=1, \ldots, \mathrm{K}
\end{gathered}
$$

où $\bar{x}_{j}^{i}$ est la dotation initiale de l'agent $i$ en actif $j$. Pour chaque contrat conditionnel (donnant droit à une unité de bien dans l'état $s$ ), le consommateur doit s'acquitter de $p_{s}$ unités de compte et de $p_{k s}$ unités de monnaie de type $k$ $(k=1, \ldots, \mathrm{K})$. En raison de la ressemblance du programme (II) avec le programme d'un consommateur en situation de rationnement, nous appellerons prix de rationnement les $p_{k s}$ et revenu de rationnement les $\varepsilon_{k}^{i}$. Rappelons en effet qu'on peut représenter le rationnement comme un système à monnaies multiples dans lequel chaque consommateur reçoit des revenus et paie des prix non seulement en numéraire traditionnel mais encore en monnaie de rationnement ${ }^{1}$. Ce système a été appliqué pendant les périodes de guerre où, pour se procurer de la viande sur le marché officiel, on devait non seulement payer avec de la monnaie fiduciaire mais encore fournir un certain nombre de points de rationnement d'une carte d'alimentation. Dans un tel système, un consommateur ne peut légalement acheter un bien s'il n'a pas suffisamment de points de rationnement de la catégorie à laquelle appartient ce bien et ce, même s'il est suffisamment riche.

$\mathrm{Si}$, par exemple, l'individu ne peut consommer dans l'état $\mathrm{S}$ et peut consommer autant qu'il le souhaite, dans la limite de son budget, dans tous les autres états, les coefficients de l'unique contrainte de rationnement seront alors égaux à $p_{1 s}=0$ pour $s \neq \mathrm{S}, p_{1 s}=1$ et $\varepsilon_{1}^{i}=0$ (ce qui est équivalent à l'équation $\mathrm{C}_{\mathrm{S}}^{i}=0$ ). Le consommateur ne pourra consommer dans l'état $S$ car, pour se faire, il devrait

1. Voir J. Tobin [1952]. 
payer une quantité de points de rationnement égale au nombre d'unités de bien de consommation désirées, alors qu'il n'a pas de points de rationnement.

On peut rendre équivalent le problème canonique de la sélection de portefeuille (programme I) et le problème du choix des biens contingents à points de rationnement (programme II) s'il est possible de déterminer $\left(\mathbf{P}, \boldsymbol{\varepsilon}^{i}\right)$ tels que $\mathbf{P} \mathbf{C}^{i}=\varepsilon^{i}$ si, et seulement si, il est possible d'obtenir $\mathbf{C}^{i}$ à partir des actifs disponibles $\mathbf{Y}$ et des dotations futures $\boldsymbol{e}^{i}$. Le théorème suivant permet de montrer qu'il est toujours possible de déterminer une telle matrice $\mathbf{P}$ et un tel vecteur $\varepsilon^{i}$.

THÉORÈME 3 (Généralisation de S. Fischer [1972]). La matrice des paiements $\mathbf{Y}$ et le vecteur des dotations $\boldsymbol{e}^{i}$ définissent de manière unique l'ensemble des possibilités de consommation pour un problème de biens contingents à points de rationnement.

En effet, on peut décomposer l'équation (9) de la manière suivante :

$\mathbf{C}_{\mathrm{N}}^{i}=\left[\mathbf{Y}_{\mathrm{N}}\right] \boldsymbol{x}^{i}+\boldsymbol{e}_{\mathrm{N}}^{i}$ où $\left[\mathbf{Y}_{\mathrm{N}}\right]$ est une matrice carrée d'ordre $\mathrm{N}$

$\mathbf{C}_{\mathrm{S}-\mathrm{N}}^{i}=\left[\mathbf{Y}_{\mathrm{S}-\mathrm{N}}\right] \boldsymbol{x}^{i}+\boldsymbol{e}_{\mathrm{S}-\mathrm{N}}^{i}$ où $\left[\mathbf{Y}_{\mathrm{S}-\mathrm{N}}\right]$ est une matrice $(\mathrm{S}-\mathrm{N} \times \mathrm{N})$

En remplaçant la valeur de $\boldsymbol{x}$ donnée par la première équation dans la seconde on obtient :

$$
\begin{gathered}
\mathbf{C}_{\mathrm{S}-\mathrm{N}}^{i}=\left[\mathbf{Y}_{\mathrm{S}-\mathrm{N}}\right]\left[\left[\mathbf{Y}_{\mathrm{N}}^{-1}\right]\left(\mathbf{C}_{\mathrm{N}}^{i}-\boldsymbol{e}_{\mathrm{N}}^{i}\right)\right]+\boldsymbol{e}_{\mathrm{S}-\mathrm{N}}^{i} \\
\mathbf{C}_{\mathrm{S}-\mathrm{N}}^{i}-\left[\mathbf{Y}_{\mathrm{S}-\mathrm{N}}\right]\left[\mathbf{Y}_{\mathrm{N}}^{-1}\right] \mathbf{C}_{\mathrm{N}}^{i}=\boldsymbol{e}_{\mathrm{S}-\mathrm{N}}^{i}-\left[\mathbf{Y}_{\mathrm{S}-\mathrm{N}}\right]\left[\mathbf{Y}_{\mathrm{N}}^{-1}\right] \boldsymbol{e}_{\mathrm{N}}^{i}
\end{gathered}
$$

ce que l'on peut réécrire de manière plus compacte :

$$
\mathbf{P} \mathbf{C}^{i}=\varepsilon^{i}
$$

où $\mathbf{P}=\left[-\left[\mathbf{Y}_{\mathrm{S}-\mathrm{N}}\right]\left[\mathbf{Y}_{\mathrm{N}^{-1}}^{-1}\right]: \mathbf{I}_{\mathrm{S}-\mathrm{N}}\right] ; \varepsilon^{i}=\mathbf{P} \boldsymbol{e}^{i}$ et $\mathbf{I}_{\mathrm{S}-\mathrm{N}}$ est la matrice identité d'ordre (S-N).

D'autre part, la richesse dont dispose le consommateur en première période est égale à la valeur présente de ses dotations en biens ( $e_{0}^{i}$ et $\boldsymbol{e}^{\boldsymbol{i}}$ ) et en actifs financiers (égales à la valeur des biens qu'il permettent d'obtenir, c-à-d $\mathbf{Y} \overrightarrow{\boldsymbol{x}}^{i}$ ) évaluée à partir du système de prix des biens contingents $(1, p)$ :

$$
e_{0}^{i}+\boldsymbol{p}^{\prime}\left(\mathbf{Y} \overline{\boldsymbol{x}}^{i}+\boldsymbol{e}^{i}\right)=e_{0}^{i}+\boldsymbol{q}^{\prime} \overline{\boldsymbol{x}}^{i}+\boldsymbol{p}^{\prime} \boldsymbol{e}^{i}
$$

On voit donc qu'en l'absence d'opportunités d'arbitrage la richesse initiale dans le problème du choix de biens contingents à points de rationnement diffère de la richesse initiale dans le problème du choix de portefeuille de :

$$
\boldsymbol{p}^{\prime} \boldsymbol{e}^{i}
$$

Cette différence s'annule lorsque les dotations futures du consommateur sont nulles. C'est le cas envisagé par Fischer. Le programme du consommateur dans l'incertain se réduit alors à celui d'un consommateur en situation de rationnement qui, pour chaque groupe de biens rationnés, devrait équilibrer ses achats et ses ventes en termes de points de rationnement. En effet, dans ce cas on a :

$$
\varepsilon^{i}=\left[-\left[\mathbf{Y}_{\mathrm{S}-\mathrm{N}}\right]\left[\mathbf{Y}_{\mathbf{N}^{-}}^{-1}\right]: \mathbf{I}_{\mathrm{S}-\mathrm{N}}\right] \mathbf{0}=\mathbf{0}
$$

Nous avons donc montré l'équivalence entre le problème canonique de la sélection de portefeuille et le problème du choix de biens contingents à points de rationnement. On constate de plus que ce dernier problème est parfaitement isomorphe au problème du choix du consommateur en situation de rationnement. Notons également que, dans le cas d'un système complet de marchés, la 
matrice des prix de rationnement est égale à la matrice nulle et l'on se ramène au cas classique du choix de biens contingents sous une contrainte de budget unique de Arrow et Debreu ${ }^{1}$.

En suivant la méthode de Luenberger, on peut faire le lien entre l'approche de Fischer [1972] et celle de He et Pearson [1991]. En effet, He et Pearson cherchent à substituer au programme d'optimisation dynamique d'un consommateur en situation d'incertitude un programme d'optimisation statique à une seule contrainte. Pour ce faire, ils doivent choisir parmi l'ensemble des vecteurs de prix d'états compatibles avec l'absence d'opportunités d'arbitrage celui qui est tel que la solution du programme statique (avec ce système particulier de prix) est identique à la solution dynamique. Ce système de prix est tel que la demande du consommateur pour les biens de consommation conditionnels qui ne peuvent être atteints par le système incomplet d'actifs financiers est nulle. He et Pearson montrent que ceci revient à choisir le système de prix qui minimise le maximum d'utilité que le consommateur peut atteindre. Il existe donc autant de systèmes de prix qu'il y a de consommateurs.

Fischer, quant à lui, cherche à substituer au programme d'optimisation dynamique un programme d'optimisation statique (à éventuellement plusieurs contraintes). Dans ce cas, les prix d'état et les prix de rationnement sont les mêmes pour tous les consommateurs et se déduisent des caractéristiques objectives des actifs (leurs prix et leurs paiements). Grâce à la méthode de Luenberger, on peut déterminer, à partir du programme du consommateur en situation de rationnement, le système de prix d'état tel que la solution du programme statique est identique à celle du programme dynamique. En effet, le programme II aux $\mathrm{K}+1$ contraintes est équivalent au programme à contrainte unique suivant :

$$
\begin{gathered}
\operatorname{Max} \sum_{s=1}^{\mathrm{S}} \pi_{s}^{i} \mathrm{U}^{i}\left(\mathrm{C}_{0}^{i}, \mathrm{C}_{s}^{i}\right) \\
\lambda_{0} \mathrm{C}_{0}^{i}+\sum_{s=1}^{\mathrm{S}}\left(\lambda_{0} p_{s}+\sum_{k=1}^{\mathrm{K}} \lambda_{k} p_{k s}\right) \mathrm{C}_{s}^{i} \leqslant \lambda_{0} e_{0}^{i}+ \\
\sum_{s=1}^{\mathrm{S}}\left(\lambda_{0} p_{s}+\sum_{k=1}^{\mathrm{K}} \lambda_{k} p_{k s}\right)\left(e_{s}^{i}+\sum_{j=1}^{\mathrm{N}} y_{j s} \bar{x}_{j}^{i}\right)
\end{gathered}
$$

On peut donc considérer que le système de prix d'état qui permet de ramener le problème dynamique à un problème statique à contrainte unique est donné par :

$$
\lambda_{0} p_{s}+\sum_{k=1}^{\mathrm{K}} \lambda_{k} p_{k s}
$$

1. Le mérite d'avoir noté l'équivalence d'un marché financier avec moins d'actifs que d'états de la nature à un marché d'actifs Arrow-Debreu à points de rationnement revient à D. Cass et J. Stiglitz [1970]. 
En effet, pour obtenir une unité de bien dans l'état $s$, le consommateur doit payer en première période (en termes du numéraire) $\lambda_{0} p_{s}$ et donner $p_{k s}$ points de rationnement de type $k(k=1, \ldots, \mathrm{K})$ dont le prix en termes du numéraire est égal à $\lambda_{k}$. L'avantage de la formulation de Fischer par rapport à la formulation dynamique c'est qu'elle permet de calculer le système de prix implicites correspondant au programme statique à contrainte unique beaucoup plus facilement puisqu'elle ne nécessite de calculer qu'un petit nombre de multiplicateurs $(\mathrm{K}<\mathrm{S})$.

\section{Un exemple}

Un exemple nous permettra de nous assurer de l'équivalence des deux problèmes. Dans l'exemple suivant, nous considérons une économie composée d'un seul actif coûtant 2 F par unité et dont les paiements sont donnés par :

$$
\mathbf{Y}=\left[\begin{array}{l}
2 \\
1
\end{array}\right]
$$

Les dotations initiales et futures de l'agent sont respectivement :

$$
\left(e_{0}^{i}, \bar{x}^{i}\right)=(3,1) \quad \boldsymbol{e}^{i}=\left[\begin{array}{l}
3 \\
4
\end{array}\right]
$$

Nous supposons également que la fonction d'utilité de la consommation est séparable et quadratique :

$$
\mathrm{U}^{i}\left(\mathrm{C}_{0}^{i}, \mathbf{C}^{i}\right)=u^{i}\left(\mathrm{C}_{0}^{i}\right)+\operatorname{Eu}^{i}\left(\tilde{\mathrm{C}}^{i}\right)
$$

où

$$
u^{\mathrm{i}}(\mathrm{C})=\mathrm{C}-0.05(\mathrm{C})^{2}
$$

et que les états de la nature sont équiprobables. Les prix d'états sont donnés par l'équation :

$$
2 p_{1}+p_{2}=2
$$

et nous utiliserons comme vecteur particulier :

$$
\boldsymbol{p}^{\prime}=[0.5,1]
$$

Comme il n'y a qu'un actif pour deux états de la nature, il y a une seule contrainte de rationnement supplémentaire. Les prix de rationnement et le nombre de points de rationnement dont dispose l'agent sont donnés par :

$$
\mathbf{P}=(-0.5,1) \varepsilon=-0.5 \times 3+1 \times 4=2.5
$$

Dans la formulation canonique, la richesse initiale est donnée par la valeur des dotations initiales en bien et en actif :

$$
3+2 \times 1=5
$$

alors que, dans la formulation « duale », la richesse initiale est donnée par la valeur des dotations initiales et futures en bien :

$$
3+0.5 \times 5+5=10.5
$$

où les dotations en biens contingents comprennent les dotations induites par la dotation initiale en actif. 
Dans la formulation « duale » du choix de consommation dans lincertain, le programme que doit résoudre le consommateur peut s'écrire de la façon suivante :

$$
\underset{\mathrm{C}_{0}, \mathbf{C}}{\operatorname{Max}}\left(\mathrm{C}_{0}-0,05 \mathrm{C}_{0}^{2}\right)+\sum_{s=1}^{2} \pi_{s}\left(\mathrm{C}_{s}-0,05 \mathrm{C}_{s}^{2}\right)
$$

s.c.

$$
\begin{gathered}
C_{0}+0,5 C_{1}+C_{2} \leqslant 10,5 \\
-0,5 C_{1}+C_{2}=2,5
\end{gathered}
$$

Les solutions optimales sont :

$$
\left(C_{0}^{*}, C_{1}^{*}, C_{2}^{*}\right)=(5,3,4)
$$

et le niveau d'utilité atteint est $\mathrm{U}^{*}=6.625$.

Dans la formulation canonique du choix de consommation dans l'incertain, le programme que doit résoudre le consommateur s'écrit :

$$
\begin{gathered}
\underset{\mathrm{C}_{0}, x}{\operatorname{Max}}\left(\mathrm{C}_{0}-0,05 \mathrm{C}_{0}^{2}\right)+\sum_{s=1}^{2} \pi_{s}\left(\mathrm{C}_{s}-0,05 \mathrm{C}_{s}^{2}\right) \\
\mathrm{C}_{0}+2 x \leqslant 5 \quad\left(\lambda_{0}\right) \\
\mathrm{C} \leqslant\left[\begin{array}{l}
2 \\
1
\end{array}\right] x+\left[\begin{array}{l}
3 \\
4
\end{array}\right] \quad\left(\begin{array}{l}
\lambda_{1} \\
\lambda_{2}
\end{array}\right)
\end{gathered}
$$

Les solutions optimales sont :

$$
\left(C_{0}^{*}, x^{*}\right)=(5,0)
$$

ce qui donne comme consommation future :

$$
\left(C_{1}^{*}, C_{2}^{*}\right)=(3,4)
$$

En appliquant la méthode de Luenberger, on peut trouver le système de prix implicites tel que le programme dynamique (ou le programme statique) à contraintes multiples peut se ramener à un programme statique à contrainte unique. En effet, la valeur des multiplicateurs à l'équilibre est donnée par :

$$
\left(\lambda_{0}^{*}, \lambda_{1}^{*}, \lambda_{2}^{*}\right)=(0,5,0,35,0,3)
$$

où les prix implicites satisfont à la condition d'absence d'opportunités d'arbitrage puisque l'actif vaut deux unités de consommation courante (et par conséquent $0.5 \times 2=1$ unité de compte) et qu'il rapporte deux unités de biens dans le premier état de la nature et une unité dans le second (ce qui équivaut à $0.35 \times 2$ $+0.3 \times 1=1$ unité de compte) ${ }^{l}$. En utilisant ces prix implicites, on peut écrire le programme statique à contrainte budgétaire unique suivant dont la solution

1. La valeur des multiplicateurs pour le programme statique à points de rationnement est donnée par $\left(\lambda_{0}^{*}, \mu^{*}\right)=(0,5,-0,2)$. Les prix implicites sont donc égaux à $p_{1}=0,5 \times$ $0,5-0,5 \times(-0,2)=0,35$ et $p_{2}=0,5-0,2=0,3$. 
est identique au programme dynamique (ou statique) à contraintes budgétaires multiples :

$$
\underset{\mathrm{C}_{0}, \mathrm{C}}{\operatorname{Max}}\left(\mathrm{C}_{0}-0,05 \mathrm{C}_{0}^{2}\right)+\sum_{s=1}^{2} \pi_{s}\left(\mathrm{C}_{s}-0,05 \mathrm{C}_{s}^{2}\right)
$$

s.c.

$$
0,5 \mathrm{C}_{0}+0,35 \mathrm{C}_{1}+0,3 \mathrm{C}_{2} \leqslant 4,75
$$

La solution de ce programme est donnée par :

$$
\left(C_{0}^{*}, C_{1}^{*}, C_{2}^{*}\right)=(5,3,4)
$$

\section{EXISTENCE ET OPTIMALITÉ DES MARCHÉS FINANCIERS INCOMPLETS}

Dans cette section, nous étudierons dans le cadre du modèle des marchés de biens conditionnels à points de rationnement le problème de la détermination de l'équilibre et de son optimalité. En effet, on peut utiliser le théorème de Fischer pour montrer que l'ensemble des allocations d'équilibre du modèle des marchés de biens conditionnels à point de rationnement est identique à l'ensemble des allocations d'équilibre du modèle des marchés financiers incomplets. Grâce au théorème de Fischer, on peut ainsi généraliser ${ }^{1}$ le résultat d'Arrow [1964] au cas des marchés financiers incomplets puisque le modèle des marchés de biens conditionnels complets de Arrow-Debreu est un cas particulier du modèle des marchés de biens conditionnels à point de rationnement de Fischer : c'est le cas particulier dans lequel il n'y a pas de contrainte de rationnement.

$$
\text { Par définition, on dit que } \begin{aligned}
\left(\left[\mathrm{C}_{0}^{i *}, \mathrm{C}_{s}^{i *}\right],\left[x_{j}^{j *}\right],\left[q_{j}^{*}\right],\left[y_{j s}\right]\right)_{i} & =1, \ldots, \mathrm{I} \\
j & =1, \ldots, \mathrm{N} \\
s & =1, \ldots, \mathrm{S}
\end{aligned} \text { est un équi- }
$$

libre à la Arrow du modèle des marchés financiers incomplets si et seulement si les conditions suivantes sont respectées :

- $\left(\left[\mathrm{C}_{0}^{i^{*}}, \mathrm{C}_{s}^{i^{*}}\right],\left[x_{j}^{i^{*}}\right]\right)_{j}=1, \ldots, \mathrm{N}$ est solution du programme $(\mathrm{I})$ pour $i=1, \ldots, \mathrm{I}$

$$
\begin{aligned}
& \sum_{i=1}^{\mathrm{I}} \mathrm{C}_{s}^{i^{*}}=\sum_{i=1}^{\mathrm{I}} e_{s}^{i} \quad s=0, \ldots, \mathrm{S} \\
& \sum_{i=1}^{\mathrm{I}} x_{j}^{i}=0 \quad j=1, \ldots, \mathrm{N}
\end{aligned}
$$

1. La généralisation n'est pas totale dans la mesure où nous avons supposé un seul bien physique. 
où nous avons supposé, pour simplifier, que les dotations initiales des agents en actifs financiers sont nulles.

Par définition, on dit que

$$
\begin{aligned}
\left(\left[\mathrm{C}_{0}^{i^{* *}}, \mathrm{C}_{s}^{j^{* *}}\right], p_{0}^{*},\left[p_{s}^{*}\right],\left[p_{s}^{k}\right]\right)_{i} & =1, \ldots, \mathrm{I} \\
k & =1, \ldots, \mathrm{K} \text { est un équi- } \\
s & =1, \ldots, \mathrm{S}
\end{aligned}
$$

libre à la Fischer du modèle des marchés de biens conditionnels à points de rationnement si, et seulement si :

$-\left(\left[\mathrm{C}_{0}^{i * *}, \mathrm{C}_{s}^{i^{* *}}\right]\right)_{s=1, \ldots, \mathrm{s}}$ est solution du programme (II) pour $i=1, \ldots, \mathrm{I}$

$-\sum_{i=1}^{\mathrm{I}} \mathrm{C}_{s}^{i^{* *}}=\sum_{i=1}^{\mathrm{I}} e_{s}^{i} \quad s=0, \ldots, \mathrm{S}$

Le théorème de Fischer permet de montrer que l'ensemble des allocations d'équilibre à la Arrow $\left(\left[\mathrm{C}_{0}^{i *}, \mathrm{C}_{s}^{i *}\right]\right)_{\substack{i=1 \\ s=1, \ldots, \mathrm{I}}}$ est identique à l'ensemble des allocations d'équilibre à la Fischer $\left(\left[\mathrm{C}_{0}^{* *}, \mathrm{C}_{s}^{i^{* *}}\right]\right)_{\substack{i=1 \\ s=1, \ldots, \mathrm{S}}}$. En effet, on sait d'après le théorème de Fischer que si $\left(\left[\mathrm{C}_{0}^{i^{*}}, \mathrm{C}_{s}^{i^{*}}\right]\right)_{\substack{i=1 \\ s=1, \ldots, \mathrm{l}}}$ est une solution du programme I lorsque les prix des actifs financiers sont donnés par $\boldsymbol{q}^{*}$ alors $\left(\left[\mathrm{C}_{0}^{i *}, \mathrm{C}_{s}^{i *}\right]\right)_{\substack{i \\ s}=1, \ldots, \mathrm{I}}$ est également solution du programme II si $p_{0}^{*}=1$ et $\left[p_{s}^{*}\right]_{s=1, \ldots, s}$ tels que $\boldsymbol{q}^{*}=\mathbf{Y}^{\prime} \boldsymbol{p}^{*}$ ainsi que $\mathbf{P}^{*}=\left[-\left[\mathbf{Y}_{\mathrm{S}-\mathrm{N}}\right]\left[\mathbf{Y}_{\mathrm{N}}^{-1}\right]: \mathbf{I}_{\mathrm{S}-\mathrm{N}}\right] . \mathrm{La}$ première condition pour qu'une allocation soit un équilibre à la Fischer est donc vérifiée. La seconde l'est également puisqu'elle est identique à la seconde condition d'un équilibre à la Arrow.

On a bien une généralisation du résultat d'Arrow [1964] puisque, lorsque les marchés financiers sont complets, il n'y a pas de contraintes de rationnement et le modèle de Fischer est équivalent au modèle d'Arrow-Debreu. Arrow [1964] s'est servi de cette équivalence pour étudier les problèmes de l'existence et de l'optimalité des allocations d'équilibre du modèle des marchés financiers complets : une allocation d'équilibre dans un tel modèle existe et est efficace au sens de Pareto si, et seulement si, une allocation correspondante dans le modèle des marchés de biens conditionnels complets existe et est efficace au sens de Pareto. Plutôt que d'étudier les conditions d'existence et d'efficacité des équilibres de marchés financiers incomplets ${ }^{1}$, nous allons étudier les conditions d'existence (en fait, la détermination) et d'optimalité des équilibres de marchés de biens conditionnels à point de rationnement. Ceci nous permettra de donner un point de vue différent sur les résultats obtenus dans le cadre du modèle des marchés financiers incomplets.

1. Ce qui a été fait de façon intensive dans les années récentes ; cf. la revue de la littérature par Geanakoplos [1990]. 


\section{Détermination de l'équilibre}

Comme nous l'avons vu, le programme canonique d'un consommateur en situation d'incertitude est équivalent au programme suivant :

$$
\operatorname{Max} \sum_{s=1}^{s} \pi_{s}^{i} \quad \mathrm{U}^{i}\left(\mathrm{C}_{0}^{i}, \mathrm{C}_{s}^{i}\right)
$$

s.c.

$$
\begin{gathered}
p_{0} \mathrm{C}_{0}^{i}+\sum_{s=1}^{\mathrm{S}} p_{s} \mathrm{C}_{s}^{i} \leqslant p_{0} e_{0}^{i}+\sum_{s=1}^{\mathrm{S}} p_{s} e_{s}^{i} \\
\sum_{s=1}^{\mathrm{S}} p_{k s} \mathrm{C}_{s}^{i}=\sum_{s=1}^{\mathrm{S}} p_{k s} e_{s}^{i} \quad k=1, \ldots, \mathrm{K}=\mathrm{S}-\mathrm{N}
\end{gathered}
$$

où nous avons supposé que le consommateur ne possède initialement aucun actif financier $\left(\bar{x}_{j}^{i}=0\right)$ et où le prix du bien de consommation en première période n'a pas été normalisé.

Ecrivons le lagrangien de ce programme :

$$
\begin{aligned}
\mathrm{L}^{i}=\sum_{s=1}^{\mathrm{S}} \pi_{s}^{i} \mathrm{U}^{i}\left(\mathrm{C}_{0}^{i}, \mathrm{C}_{s}^{i}\right)+\phi_{0}^{i}\left(p_{0}\left(e_{0}^{i}-\mathrm{C}_{0}^{i}\right)\right. & \left.+\sum_{s=1}^{\mathrm{S}} p_{s}\left(e_{s}^{i}-\mathrm{C}_{s}^{i}\right)\right) \\
& +\sum_{k=1}^{\mathrm{S}-\mathrm{N}} \phi_{k}^{i} \sum_{s=1}^{\mathrm{S}} p_{k s}\left(\mathrm{C}_{s}^{i}-e_{s}^{i}\right)
\end{aligned}
$$

Les demandes optimales de biens dans chaque état de la nature doivent respecter les conditions suivantes :

$$
\begin{gathered}
\frac{\partial \mathrm{L}^{i}}{\partial \mathrm{C}_{0}^{i}}=0=\sum_{s=1}^{\mathrm{S}} \pi_{s}^{i} \frac{\partial \mathrm{U}_{i}\left(\mathrm{C}_{0}^{i}, \mathrm{C}_{s}^{i}\right)}{\partial \mathrm{C}_{0}^{i}}-\phi_{0}^{i} p_{0} \\
\frac{\partial \mathrm{L}^{i}}{\partial \mathrm{C}_{s}^{i}}=0=\pi_{s}^{i} \frac{\partial \mathrm{U}_{i}\left(\mathrm{C}_{0}^{i}, \mathrm{C}_{s}^{i}\right)}{\partial \mathrm{C}_{s}^{i}}-\phi_{0}^{i} p_{s} \sum_{k=1}^{\mathrm{S}-\mathrm{N}} \phi_{k}^{i} p_{k s} \quad s=1, \ldots, \mathrm{S} \\
\frac{\partial \mathrm{L}^{i}}{\partial \phi_{0}}=0=p_{0}\left(e_{0}^{i}-\mathrm{C}_{0}^{i}\right)+\sum_{s=1}^{\mathrm{s}} p_{s}\left(e_{s}^{i}-\mathrm{C}_{s}^{i}\right) \\
\frac{\partial \mathrm{L}^{i}}{\partial \phi_{k}^{i}}=0=\sum_{s=1}^{\mathrm{S}} p_{k s}\left(\mathrm{C}_{s}^{i}-e_{s}^{i}\right) \quad k=1, \ldots, \mathrm{S}-\mathrm{N}
\end{gathered}
$$

Il existe $\mathrm{S}+1$ conditions d'équilibre :

$$
\sum_{i=1}^{1} \mathrm{C}_{0}^{i}=\sum_{i=1}^{1} e_{0}^{i}
$$




$$
\sum_{i=1}^{1} \mathrm{C}_{s}^{i}=\sum_{i=1}^{\mathrm{I}} e_{s}^{i} \quad s=1, \ldots, \mathrm{S}
$$

Comme il y a $\mathrm{S}-\mathrm{N}+1$ contraintes budgétaires par consommateur il y a $\mathrm{S}-\mathrm{N}+1$ lois de Walras :

$$
\begin{aligned}
& p_{0}\left(\sum_{i=1}^{\mathrm{I}} \mathrm{C}_{0}^{i}-\sum_{i=1}^{\mathrm{I}} e_{0}^{i}\right)+\sum_{s=1}^{\mathrm{S}} p_{s}\left(\sum_{i=1}^{\mathrm{I}} \mathrm{C}_{s}^{i}-\sum_{i=1}^{\mathrm{I}} e_{s}^{i}\right)=0 \\
& \sum_{s=1}^{\mathrm{S}} p_{k s}\left(\sum_{i=1}^{\mathrm{I}} \mathrm{C}_{2 s}^{i}-\sum_{i=1}^{\mathrm{I}} e_{s}^{i}\right)=0 \quad k=1, \ldots, \mathrm{D}
\end{aligned}
$$

où le degré d'incomplétude est égal à $\mathrm{D}=\mathrm{S}-\mathrm{N}$.

$\mathrm{D}^{\prime}$ après le théorème des fonctions implicites ${ }^{1}$, un système de $\mathrm{J}$ équations à $\mathrm{K}$ variables $(J \leqslant K)$ permet d'exprimer localement $\mathbf{J}$ variables comme des fonctions différentiables des $\mathbf{K}-\mathbf{J}$ autres variables.

Il existe $(S+1)+I \times\{1+S+1+(S-N)\}$ équations: $(S+1)$ équations d'équilibre de marché et $\{1+\mathrm{S}+1+(\mathrm{S}-\mathrm{N})\}$ conditions d'optimalité par consommateur. En tenant compte des lois de Walras il reste $(S+1)+I \times$ $\{1+S\}+(I-1) \times\{S-N+1\}$ équations indépendantes.

Si l'on suppose que les prix de rationnement sont prédéterminés, il y a $(S+1)$ $+I \times\{1+S\}+I \times\{1+(S-N)\}$ variables endogènes: $(S+1)$ prix, $(S+1)$ demandes par consommateur et $(S-N+1)$ multiplicateurs de Lagrange par consommateur. Il y a donc en tout $(\mathrm{S}-\mathrm{N}+1)$ inconnues de plus que d'équations et l'on devra exprimer $(S+1)+I \times\{1+S\}+(I-1) \times\{S-N+1\}$ variables en fonction de $(S-N+1)$ autres choisies arbitrairement comme paramètre. $\mathrm{On}$ dira donc qu'il y a (D+1) degrés d'indétermination nominale.

La question se pose de savoir si cette indétermination nominale est significative, c'est-à-dire si les valeurs réelles sont affectées par les paramètres. Autrement dit, les quantités de biens consommés dépendent-elles de la valeur des paramètres ? Si oui, on parlera d'indétermination réelle. Dans le cas où les prix de rationnement sont prédéterminés (ce qui correspond à l'hypothèse d'exogénéité du paiement des actifs), il y a un seul degré d'homogénéité. En effet, si $\begin{aligned} &\left(\left[\mathrm{C}_{0}^{i^{*}}, \mathrm{C}_{s}^{i^{*}}\right], p_{0}^{*},\left[p_{s}^{*}\right],\left[\phi_{0}^{i *}, \phi_{k}^{i^{*}}\right],\left[\bar{p}_{k s}\right]\right)_{i}=1, \ldots, \mathrm{I} \\ & k=1, \ldots, \mathrm{S}-\mathrm{N} \\ & s=1, \ldots, \mathrm{S}\end{aligned}$

formé par les équations d'équilibre de marché et les conditions d'optimalité des consommateurs alors

$$
\begin{aligned}
\left(\left[\mathrm{C}_{0}^{i^{*}}, \mathrm{C}_{s}^{i^{*}}\right], \mu \times p_{0}^{*},\left[\mu \times p_{s}^{*}\right],\left[\phi_{0}^{i^{*}} / \mu, \phi_{k}^{*}\right],\left[\bar{p}_{k s}\right]\right)_{i}=1, \ldots, \mathrm{I} \\
k=1, \ldots, \mathrm{S}-\mathrm{N} \\
s=1, \ldots, \mathrm{S}
\end{aligned}
$$

solution de ce même système d'équations. On peut ainsi fixer arbitrairement la valeur d'un prix, par exemple le prix du bien de consommation en première

1. Voir D. Cass [1990], p. 21. 
période. Il reste $(S+1)+I \times\{1+S\}+(I-1) \times\{S-N+1\}$ inconnues que l'on doit exprimer en fonction de $(S-N)$ paramètres. Il y a donc $D$ degrés d'indétermination réelle.

Si l'on suppose que les prix de rationnement ne sont pas prédéterminés (ce qui correspond à l'hypothèse d'endogénéité des paiements des actifs), ceci revient à ajouter $(S-N) \times S$ inconnues. En effet, il y a $(S-N)$ prix de rationnement par état de la nature. Il y a donc $\mathbf{D} \times(\mathbf{S}+\mathbf{1})+\mathbf{1}$ degrés d'indétermination nominale. Par ailleurs il existe $(S-N+1)$ degrés d'homogénéité. En effet si

$$
\begin{aligned}
\left(\left[\mathrm{C}_{0}^{i *}, \mathrm{C}_{s}^{i^{*}}\right], p_{0}^{*},\left[p_{s}^{*}\right],\left[\phi_{0}^{i^{*}}, \phi_{k}^{i^{*}}\right],\left[p_{k s}^{*}\right]\right)_{i} & =1, \ldots, \mathrm{I} \\
k & =1, \ldots, \mathrm{s}-\mathrm{N} \\
s & =1, \ldots, \mathrm{S}
\end{aligned}
$$

formé par les équations d'équilibre de marché et les conditions d'optimalité des consommateurs alors

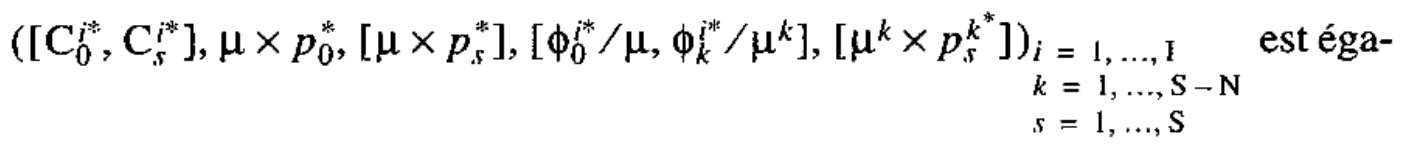

lement solution de ce même système d'équations. On peut ainsi fixer arbitrairement la valeur de $(S-N+1)$ prix, par exemple le prix du bien de consommation en première période et les prix de rationnement du premier état de la nature. Il y a donc $\mathbf{D} \times \mathbf{S}$ degrés d'indétermination réelle.

On remarque que l'indétermination nominale ou réelle est indépendante du nombre d'agents économiques et qu'elle est d'autant plus forte que le degré d'incomplétude est grand. Par ailleurs, que les prix de rationnement soient prédéterminés ou pas lorsque le degré d'incomplétude est nul, il n'y a pas d'indétermination réelle et un seul degré d'indétermination nominale. On peut alors déterminer $\mathrm{S}$ prix. Par exemple, on pourra normaliser le prix du bien en première période et les conditions d'équilibre détermineront les prix d'état. On retrouve le résuitat classique de l'équilibre (walrasien) dans un système de marchés complets où le niveau général des prix est indéterminé.

\section{Optimalité}

On sait qu'on peut faire correspondre à chaque allocation optimale au sens de Pareto un ensemble de nombres non négatifs, $\left(\lambda^{i}\right)_{i=1}^{I}$, tel que la même allocation puisse être réalisée par un planificateur social maximisant sous contrainte de ressources une combinaison linéaire des fonctions d'utilité individuelles en prenant comme poids les coefficients $\left(\lambda^{i}\right)_{i=1}^{\mathrm{I}}$ :

$$
\operatorname{Max} \sum_{i=1}^{\mathrm{I}} \lambda^{i}\left[\sum_{s=1}^{\mathrm{S}} \pi_{s}^{i} \mathrm{U}^{i}\left(\mathrm{C}_{0}^{i}, \mathrm{C}_{s}^{i}\right)\right]
$$

$$
\text { (II) } \begin{aligned}
& \sum_{i=1}^{\mathrm{I}} \mathrm{C}_{s}^{i}=e_{s} \quad \forall s=1, \ldots, \mathrm{S} \\
\sum_{i=1}^{\mathrm{I}} \mathrm{C}_{0}^{i} & =e_{0}
\end{aligned}
$$


On montre alors qu'un plan de consommation contingent faisable est une allocation optimale au sens de Pareto si, et seulement si, pour chaque état de la nature les taux marginaux de substitution sont égaux pour tous les consommateurs ${ }^{1}$.

Les conditions du premier ordre du programme (II) d'un consommateur en situation de rationnement s'écrivent :

$$
\begin{gathered}
\sum_{s=1}^{\mathrm{s}} \pi_{s}^{i} \frac{\partial \mathrm{U}_{i}\left(\mathrm{C}_{0}^{i}, \mathrm{C}_{s}^{i}\right)}{\partial \mathrm{C}_{0}^{i}}=\lambda^{i} p_{0} \\
\pi_{s}^{i} \frac{\partial \mathrm{U}_{i}\left(\mathrm{C}_{0}^{i}, \mathrm{C}_{s}^{i}\right)}{\partial \mathrm{C}_{s}^{i}}=\lambda^{i} p_{s}+\sum_{k=1}^{\mathrm{D}} \mu_{k}^{i} p_{k s}
\end{gathered}
$$

et le taux marginal de substitution entre consommation présente et future nous est donné en faisant le rapport des équations (19) et (20):

$$
\frac{\pi_{s}^{i} \frac{\partial \mathrm{U}_{i}\left(\mathrm{C}_{0}^{i}, \mathrm{C}_{s}^{i}\right)}{\partial \mathrm{C}_{s}^{i}}}{\sum_{s=1}^{\mathrm{s}} \pi_{s}^{i} \frac{\partial \mathrm{U}_{i}\left(\mathrm{C}_{0}^{i}, \mathrm{C}_{s}^{i}\right)}{\partial \mathrm{C}_{0}^{i}}}=p_{s} / p_{0}+\sum_{k-1}^{\mathrm{D}}\left(\mu_{k}^{i} / \lambda^{i}\right)\left(p_{k s} / p_{0}\right)
$$

Dans ces conditions, une allocation faisable n'est pas optimale au sens de Pareto puisque les taux marginaux de substitution dépendent des individus. Toutefois, s'il existe un marché pour les points de rationnement, les conditions du premier ordre s'écrivent :

$$
\begin{gathered}
\sum_{s=1}^{\mathrm{s}} \pi_{s}^{i} \frac{\partial \mathrm{U}_{i}\left(\mathrm{C}_{0}^{i}, \mathrm{C}_{s}^{i}\right)}{\partial \mathrm{C}_{0}^{i}}=\lambda^{i} p_{0} \\
\pi_{s}^{i} \frac{\partial \mathrm{U}_{i}\left(\mathrm{C}_{0}^{i}, \mathrm{C}_{s}^{i}\right)}{\partial \mathrm{C}_{s}^{i}}=\lambda^{i}\left(p_{s}+\sum_{k=1}^{\mathrm{D}} \alpha^{k} p_{k s}\right) \\
p_{0} \mathrm{C}_{0}^{i}+\sum_{s=1}^{\mathrm{s}}\left(p_{s}+\sum_{k=1}^{\mathrm{D}} \alpha^{k} p_{k s}\right) \mathrm{C}_{s}^{i}=p_{0} e_{0}^{i}+\sum_{s=1}^{\mathrm{s}}\left(p_{s}+\sum_{k=1}^{\mathrm{D}} \alpha^{k} p_{k s}\right) e_{s}^{i}
\end{gathered}
$$

où $\alpha^{k}$ est le prix monétaire d'un point de rationnement dans la catégorie $k$ et le taux marginal de substitution s'écrit :

$$
\mathrm{TMS}_{i}=\left(p_{s} / p_{0}+\sum_{k=1}^{\mathrm{D}} \alpha^{k}\left(p_{k s} / p_{0}\right)\right)
$$

À l'équilibre les taux marginaux de substitution de tous les consommateurs sont égaux. Cependant, il ne s'ensuit pas nécessairement que l'allocation d'équilibre est optimale au sens de Pareto. En effet, les contraintes de rationnement

1. Voir C.-F. Huang et R. Litzenberger [1988]. 
peuvent empêcher les agents d'atteindre certaines allocations optimales au sens de Pareto.

Supposons maintenant que le planificateur soit forcé d'utiliser les points de rationnement pour atteindre une allocation donnée. Il devra donner à chaque consommateur un certain nombre de points de rationnement qui leur permettront d'atteindre la meilleure configuration de consommation possible. On parle alors d'efficacité contrainte ou efficacité au sens de Diamond. Une allocation sera alors optimale au sens de Diamond si, et seulement si, elle est solution du programme suivant :

(III) s.c.

$$
\begin{array}{cc}
\underset{\left(\mathrm{C}_{t}^{i}, \varepsilon_{k}^{i}\right)}{\operatorname{Max}} \sum_{i=1}^{\mathrm{I}} \lambda_{i}\left[\sum_{s=1}^{\mathrm{S}} \pi_{s}^{i} \mathrm{U}^{i}\left(\mathrm{C}_{0}^{i}, \mathrm{C}_{s}^{i}\right)\right] \\
\sum_{i=1}^{\mathrm{I}} \mathrm{C}_{t}^{i}=e_{t} & \forall t=0, \ldots, \mathrm{S} \\
\sum_{i=1}^{\mathrm{I}} \varepsilon_{k}^{i}=\varepsilon_{k} & \forall k=1, \ldots, \mathrm{S}-\mathrm{N} \\
\sum_{S=1}^{\mathrm{S}} p_{k s} \mathrm{C}_{s}^{i}=\varepsilon_{k}^{i} & \forall i=1, \ldots, \mathrm{I} \\
\forall k=1, \ldots, \mathrm{S}-\mathrm{N}
\end{array}
$$

où $e_{t}$ représente les ressources globales disponibles dans l'état de la nature $t$ (ou à la date 0 ) et où $\varepsilon_{k}$ représente les dotations globales en point de rationnement de type $k$. Le lagrangien de ce programme s'écrit :

$$
\begin{aligned}
\mathrm{L}=\sum_{i=1}^{\mathrm{I}} \lambda^{i}\left[\sum_{s=1}^{\mathrm{S}} \pi_{s}^{i} \mathrm{U}^{i}\left(\mathrm{C}_{0}^{i}, \mathrm{C}_{s}^{i}\right)\right]+\sum_{t=0}^{\mathrm{S}} \phi_{s}\left[e_{t}-\sum_{i=1}^{\mathrm{I}} \mathrm{C}_{t}^{i}\right]+ \\
+\sum_{k=1}^{\mathrm{S}-\mathrm{N}} \gamma_{k}\left[\varepsilon_{k}-\sum_{i=1}^{\mathrm{I}} \varepsilon_{k}^{i}\right]+\sum_{i=1}^{\mathrm{I}} \sum_{k=1}^{\mathrm{S}-\mathrm{N}} \gamma_{i k}\left[\varepsilon_{k}^{i}-\sum_{s=1}^{\mathrm{S}} p_{k s} C_{s}^{i}\right]
\end{aligned}
$$

Les conditions du premier ordre s'écrivent :

$$
\begin{gathered}
\lambda^{i} \sum_{s=1}^{\mathrm{S}} \pi_{s}^{i} \frac{\partial \mathrm{U}_{i}\left(\mathrm{C}_{0}^{i}, \mathrm{C}_{s}^{i}\right)}{\partial \mathrm{C}_{0}^{i}}=\phi_{0} \quad i=1, \ldots, \mathrm{I} \\
\lambda^{i} \pi_{s}^{i} \frac{\partial \mathrm{U}_{i}\left(\mathrm{C}_{0}^{i}, \mathrm{C}_{s}^{i}\right)}{\partial \mathrm{C}_{s}^{i}}=\phi_{s}+\sum_{k=1}^{\mathrm{S}-\mathrm{N}} \gamma_{i k} p_{k s} \quad \begin{array}{l}
i=1, \ldots, \mathrm{I} \\
s=1, \ldots, \mathrm{S}
\end{array} \\
\sum_{i=1}^{\mathrm{I}} \mathrm{C}_{t}^{i}=e_{t} \quad t=0, \ldots, \mathrm{S} \\
\gamma_{i k}=\gamma_{k} \quad \begin{array}{l}
i=1, \ldots, \mathrm{I} \\
k=1, \ldots, \mathrm{S}-\mathrm{N}
\end{array}
\end{gathered}
$$


Cette dernière condition est particulièrement intéressante puisqu'elle signifie que si pour un individu particulier une contrainte de rationnement ne l'empêche pas d'atteindre une allocation particulière il en sera de même pour tous les individus. De plus, la demande globale sur ce marché de points de rationnement sera inférieure à l'offre globale.

En faisant le rapport des équations (26) et (27) et en substituant $\gamma_{k}$ à $\gamma_{i k}$ on obtient le taux marginal de substitution de la consommation présente à la consommation conditionnelle :

$$
\frac{\pi_{s}^{i} \frac{\partial \mathrm{U}_{i}\left(\mathrm{C}_{0}^{i}, \mathrm{C}_{s}^{i}\right)}{\partial \mathrm{C}_{s}^{i}}}{\sum_{s=\mathrm{I}}^{\mathrm{s}} \pi_{s}^{i} \frac{\partial \mathrm{U}_{i}\left(\mathrm{C}_{0}^{i}, \mathrm{C}_{s}^{i}\right)}{\partial \mathrm{C}_{0}^{i}}}=\phi_{s} / \phi_{0}+\sum_{k=1}^{\mathrm{D}} \gamma_{k}\left(p_{k s} / \phi_{0}\right)
$$

On constate qu'une allocation est efficace au sens de Diamond si, et seulement si, les taux marginaux de substitution sont identiques pour tous les consommateurs. La comparaison des équations (25) et (30) nous permet donc d'affirmer que l'ouverture d'un marché où les consommateurs ont la possibilité d'échanger les points de rationnement contre du numéraire conduit à une allocation optimale au sens de Diamond. Il est alors impossible d'augmenter l'utilité d'un consommateur rationné sans diminuer celle d'un autre.

La différence entre les taux marginaux de substitution en l'absence de rationnement et les taux marginaux de substitution en présence de rationnement est égale à :

$$
\sum_{k=1}^{\mathrm{D}} \gamma_{k}\left(p_{k s,} / \phi_{0}\right)
$$

L'allocation est efficace au sens de Pareto si l'une des deux conditions suivantes est vérifiée :

$$
\begin{array}{ll}
p_{k s}=0 & \forall k=1, \ldots, \mathrm{S}-\mathrm{N} \text { et } \forall s=1, \ldots, \mathrm{S} \\
\gamma_{k}=0 & \forall k=1, \ldots, \mathrm{S}-\mathrm{N}
\end{array}
$$

Dans ces cas, les taux marginaux de substitution en situation de rationnement sont égaux aux taux marginaux de substitution en l'absence de rationnement. La première condition signifie qu'on est dans une situation de marché complet (absence de rationnement), alors que la seconde condition signifie que le rationnement n'est pas contraignant. En effet, d'après le théorème de Kuhn et Tucker, on a dans ce cas :

$$
\sum_{s=1}^{s} p_{k s} C_{s}^{i}<\varepsilon_{k}^{i} \Rightarrow \gamma_{i k}=0=\gamma_{k}
$$

Le rationnement n'empêche pas les agents économiques d'atteindre le plan de consommation qu'ils désirent. Les marchés sont incomplets mais cette incomplétude est redondante dans la mesure où les allocations qui ne peuvent être atteintes à cause du rationnement ne sont pas efficaces. Dans les deux cas, il sera possible d'atteindre une allocation efficace au sens de Pareto. 
Une allocation optimale au sens de Pareto peut être atteinte même lorsque les marchés sont incomplets lorsque les agents ont suffisamment de points de rationnement pour atteindre cette allocation particulière. En effet, soit $\left(\left[\mathrm{C}_{0}^{i *}, \mathrm{C}_{s}^{i^{*}}\right]\right)_{s=1, \ldots, \mathrm{s}}$ une allocation optimale au sens de Pareto. Puisqu'il est possible de donner aux agents suffisamment de points de rationnement pour atteindre cette allocation particulière, cela signifie que pour tout consommateur, et quelle que soit la contrainte de rationnement, on a :

$$
\sum_{s=1}^{\mathrm{s}} p_{k s} \mathrm{C}_{s}^{i^{*}}<\varepsilon_{k}^{i}
$$

Les multiplicateurs associés à chaque contrainte individuelle de rationnement $\gamma_{i k}$ sont nuls ainsi que les multiplicateurs associés à l'équilibre global de chaque marché de rationnement $\gamma_{k}$ (puisqu'à l'optimum d'efficacité contrainte ces deux multiplicateurs doivent être égaux).

Si la fonction d'utilité est séparable et qu'il est possible d'atteindre certains éléments d'un vecteur de consommation efficace au sens de Pareto, alors ces mêmes éléments seront constitutifs d'un vecteur de consommation efficace au sens de Diamond.

Notons enfin que si le planificateur a le pouvoir de fixer les prix de rationnement, il les fixera égaux à zéro. En effet, la condition d'optimalité pour les prix de rationnement est donnée par:

$$
\frac{\partial \mathrm{L}}{\partial p_{k s}}=-\sum_{i=1}^{\mathrm{I}} \gamma_{i k} \mathrm{C}_{s}^{i}=-\gamma_{k} \sum_{i=1}^{\mathrm{I}} \mathrm{C}_{s}^{i}
$$

La consommation des agents étant non négative, la dérivée partielle du Lagrangien par rapport aux prix de rationnement est strictement négative, ce qui implique que les prix de rationnement optimaux sont nuls. Le résultat est en accord avec l'intuition puisque, si tous les prix de rationnement sont nuls, il n'y a plus de contraintes de rationnement et, par conséquent, il est possible d'atteindre une allocation efficace au sens de Pareto, ce qui est évidemment préférable à une allocation contrainte.

\section{CONCLUSION}

C'est une constante dans l'histoire de la théorie économique contemporaine qu'un grand nombre de progrès significatifs ont été permis par le remplacement de modèles descriptivement réalistes mais analytiquement impossibles à utiliser par des modèles irréalistes, quoique équivalents aux premiers mais analytiquement plus faciles à exploiter. Le point de vue que nous avons voulu défendre dans cet article, c'est que le modèle des marchés de biens conditionnels à point de rationnement de Fischer [1972] doit être ajouté au nombre de ces progrès significatifs.

L'existence d'un isomorphisme peut permettre de réconcilier l'irréalisme des hypothèses avec la conformité aux faits des prédictions du modèle. Un modèle reposant sur des hypothèses irréalistes peut rendre compte malgré tout des phé- 
nomènes économiques parce qu'il existe un modèle réaliste parfaitement isomorphe ${ }^{1}$.

En effet, il existe deux catégories de modèles irréalistes :

1. Les modèles irréalistes «par défaut » qui font abstraction de certains aspects de la réalité ;

2. Les modèles irréalistes « par excès » qui introduisent dans la modélisation des caractéristiques qui n'existent pas dans la réalité (par exemple, les biens conditionnels à points de rationnement).

On justifie l'utilisation de modèles irréalistes par défaut lorsque leurs prédictions sont conformes aux observations du monde réel auquel cas les aspects de la réalité qui ont été négligés étaient effectivement négligeables. Cependant, il existe toujours un certain malaise à utiliser des modèles irréalistes par excès même si leurs prédictions ne sont pas falsifiées. L'existence d'un isomorphisme permet d'ôter ces scrupules ainsi que d'expliquer pourquoi, paradoxalement, un modèle dont certaines des hypothèses sont contraires à la réalité peut permettre de rendre compte de cette réalité. C'est qu'il existe un modèle irréaliste par défaut isomorphe au modèle irréaliste par excès et qui retient les caractéristiques essentielles du monde réel et néglige ses caractéristiques secondaires. L'utilisation du modèle irréaliste par excès se justifiant par sa plus grande simplicité.

L'intérêt du modèle de biens conditionnels à point de rationnement de Fischer [1972], c'est qu'il permet de traiter de façon unitaire les problèmes d'indétermination et d'inefficacité de l'équilibre que l'on rencontre dans un grand nombre de modèles contemporains (par exemple, le modèle des marchés financiers incomplets et le modèle à générations imbriquées). Il permet de mettre en lumière la cause profonde de l'indétermination et de l'inefficacité. L'indétermination réelle et l'inefficacité de l'équilibre résultent de l'absence de certains marchés puisque ces problèmes disparaissent avec les contraintes de rationnement. Or, on retrouve ces problèmes d'indétermination et d'inefficacité dans de nombreux autres modèles où des contraintes pèsent de façon significative sur les échanges. Dans le cas des modèles à générations imbriquées, les générations présentes ne peuvent échanger directement avec les générations futures. Dans le cas des marchés financiers incomplets, les consommateurs ne peuvent atteindre certaines configurations de consommation désirées car l'incomplétude des marchés financiers ne leur permet pas de transférer suffisamment de pouvoir d'achat dans certains états de la nature.

Le prolongement naturel du présent article devrait être de chercher à montrer à quelle classe de modèles le modèle de Fischer est équivalent. Il serait alors possible d'étudier dans un cadre unique les questions traditionnelles relatives à l'existence, l'unicité, la stabilité et l'optimalité de l'équilibre pour toute une classe de modèles (qu'on espère la plus large possible). On retrouverait ainsi la belle unité de la théorie de l'équilibre général que permettait naguère le modèle Arrow-Debreu.

1. Cf. P. Salmon [1976]. 


\section{RÉFÉRENCES BIBLIOGRAPHIQUES}

ARROw K. [1964], « The Role of Securities in the Optimal Allocation of Risk-Bearing », Review of Economic Studies, p. 91-96.

BierwaG G., Grove M. A. [1968], « Slutsky Equations for Assets », Journal of Political Economy, p. 114-126.

CASS D. [1990], « Real Indeterminacy from Imperfect Financial Markets: Two addenda », Document de travail, CEPREMAP.

CAss D., STIGLITZ J. [1970], « The Structure of Investor Preferences and Asset Returns, and Separability in Portfolio Allocation: a Contribution to the Pure Theory of Mutual Funds », Journal of Economic Theory, p. 122-160.

CHIAPPORI P.-A. [1990], « La théorie du consommateur est-elle réfutable ? », Revue économique, p. 1001-1025.

Dalal A. J. [1983], « Comparative Statics and Asset Substituability/Complementarity in a Portfolio Model: a Dual Approach », Review of Economic Studies, p. 355-367.

Deaton A., Muellbauer J. [1980], Economics and Consumer Behaviour, Cambridge University Press, Cambridge.

FISCHER S. [1972], «Assets, Contingent Commodities, and the Slutsky Equations », Econometrica, p. 371-385.

GEANAKOPLOS J. [1990], «An Introduction to General Equilibrium with Incomplete Asset Markets ", Journal of Mathematical Economics, p. 1-38.

He H., Pearson N. D. [1991], «Consumption and Portfolio Policies with Incomplete Markets: The Infinite-Dimensional Case », Journal of Economic Theory, 54, p. 259305.

HICKS J. R. [1935], «A Suggestion for Simplifying the Theory of Money », p. 61-82, dans Critical Essays in Monetary Theory, Oxford, Clarendon Press, 1967.

Hicks J. R., Allen R. G. D. [1934], «A Reconsideration of the Theory of Value », Economica, p. 52-76 et 196-219.

HuANG C.-F., LiTZENBERGER R. H. [1988], Foundations for Financial Economics, Amsterdam, North-Holland.

INGERSOLL J. [1987], Theory of Financial Decision Making, Totowa, Rowman and Littlefield.

JARROW R. A. [1988], Finance Theory, Englewood Cliffs, Prentice-Hall.

KoLM S.-C. [1966], Les choix financiers et monétaires, Paris, Dunod.

Koulikov L. [1979], Algèbre et théorie des nombres, Moscou, Éditions Mir.

LEVY H. [1973] «The Demand For Assets Under Conditions of Risk », Journal of Finance, p. 79-96.

LUENBERGER D. [1995], Microeconomic Theory, New York, McGraw-Hill.

MAES I. [1991], « On the Origins of Portfolio Theory », Kyklos, p. 3-18.

MaLinVAUd E. [1993], Équilibre général dans les économies de marché, Paris, Economica.

Markowitz H. [1959], Portfolio Selection. Efficient Diversification of Investments, New York, Wiley.

MORISHIMA M. [1973], Theory of Demand: Monetary and Real, Cambridge, Cambridge University Press.

OHLSON J. A. [1987], The Theory of Financial Markets and Information, New York, North-Holland.

RoBBINS L. [1932], An Essay on the Nature and Significance of Economic Science, Londres, Macmillan. 
Royama S., Hamada K., [1967], « Substitution and Complementarity in the Choice of Risky Assets », dans Tobin J. et Hester D., Risk Aversion and Portfolio Choice, New York, Wiley.

Ross S. A. [1978], «A Simple Approach to the Valuation of Risky Streams », Journal of Business, p. 453-475.

SALMON P. [1976], « La méthode hypothético-déductive et les raisonnements en termes de comme si en économique. Illustration par la théorie du marché financier », Revue d'économie politique, p. 765-794.

SAMUELSON P. A. [1970], « The Fundamental Approximation Theorem of Portfolio Analysis in Terms of Means, Variances and Higher Moments », Review of Economic Studies, p. 537-542.

SANDMO A. [1969], " Capital Risk, Consumption and Portfolio Choice », Econometrica, p. $586-599$.

TOBIN J. [1952], «A Survey of the Theory of Rationning », Econometrica, p. 521-553.

TOBIN J. [1958], « Liquidity Preference as Behaviour Towards Risk », Review of Economic Studies, p. 65-86.

TSIANG S. C. [1972], «The Rationale of the Mean-Standard Deviation Analysis, Skewness Preference, and the Demand for Money ", American Economic Review, p. $442-450$.

VARIAN H. R. [1988], «Le principe d'arbitrage en économie financière », Annales d'économie et de statistique, p. 1-22. 